\title{
A Performance based Routing Classification in Cognitive Radio Networks
}

\author{
Sunita S. Barve \\ Bharati Vidyapeeth Deemed University \\ College of Engineering, Pune - 411043, India.
}

\author{
Parag Kulkarni \\ EKLaT Research \\ Pune, India
}

\begin{abstract}
Cognitive Radio Networks (CRN) is offering tremendous performance and operational benefit by providing high bandwidth to mobile users via dynamic spectrum access techniques. In this paper, we address the problem of routing in CRN which concerns about identification and maintenance of the optimal path from source to destination through intermediate relay nodes and spectrum on each link using available common channels. In this survey, the characteristics features and limiting factors of existing routing protocols are thoroughly investigated with its performance evaluation criteria's. First, the overview of the routing with its unique challenges is given under the restriction of interference and fairness to increase overall network throughput. Next, a detailed classification of the routing strategies is given according to performance evaluation matrices which are considered according to specific demand and requirements of network users. A representative selection of these strategies is discussed in detail in this paper together with services given to unique challenges of CRN. Important issues and future directions are also discussed, while highlighting the need of close coupling between interaction of network users and dynamic decision theories.
\end{abstract}

\section{General Terms}

Design, Algorithms, Management, Performance

\section{Keywords}

Wireless Network, Routing, Cognitive Radio Networks (CRN), Multi-hop Networks, Spectrum utilization, Dynamic Spectrum Utilization.

\section{INTRODUCTION}

Radio spectrum is one of the most heavily used and expensive natural resource around the world, regulated by governmental agencies. Most of the prime spectrum bands are licensed on long term basis over vast geographical regions. Recent studies indicate that many portions of the licensed spectrum are not used for a significant amount of time or in certain geographical areas. Moreover wide range of application operating in unlicensed band like ISM causes overcrowding in this band. It is becoming increasingly difficult to find spectrum that can be made available either for new services or to expand existing one.

Federal Communication Commission started new ways of managing RF resource. To that end they proposed new policy using Cognitive Radio (CR) [1] which is a promising solution to scarce spectrum resource. This will enable unlicensed/secondary users (SU) to sense and intelligently access the unoccupied spectrum portions that are not used by the licensed/primary users (PU) at any specific time.
$\mathrm{CR}$ is a radio that can change its transmitter parameters such as spectrum, modulation, and transmission power etc, based on interaction with the environment in which it operates. This can be used to sense wide range of spectrum for identifying unused spectrum holes for data communication by unlicensed users. Section II and III describes the cognitive radio network and routing in detail respectively. Categorization of routing strategies is represented in section IV according to its performance criteria's. Section V, VI and VII highlight few representative selections of these routing strategies. Important and dominant issues with future directions are discussed in section VIII followed by conclusion in section IX.

\section{COGNITIVE RADIO NETWORK}

Cognitive Radio Network (CRN) is a network in which each node is equipped with Cognitive Radio [2] who is aware of the dynamic environment and adaptively adjusts their operating parameters based on the interaction with the environment and other users of the network. These networks are offering tremendous performance and operational benefit by providing high bandwidth to mobile users via dynamic spectrum access techniques. CRN's however imposes several research challenges due to broad range of available spectrum and diverse quality of service requirement of applications.

Most of the recent research on CRN was concentrated on physical and Medium Access Control layer focusing on efficient spectrum sensing, management and sharing technique. In addition to all these unique challenges routing is also important challenge for realization of the CRN especially in the networks with multi-hop communication requirement. The design goal of Multi-hop Cognitive Radio requires the integration of cognitive principle into the rules of interaction between nodes in the network. The set of wireless nodes should form a social network that must be modelled and analysed as one entity in order to optimize the design of network functions such as resource management and routing.

In Multi-hop CRN, cognitive nodes sense wide range of spectrum and get spectrum holes and then use specific policy to select one candidate from them. The set of available channel from node to node are not static and different from one hop to another hop. Moreover existence of multiple users (Primary or Secondary) and their varying demands of transmission have great impact on path selection in routing.

Therefore traditional routing algorithms will be inefficient to handle dynamism of CRN. In CRN Quality of the end-to-end 


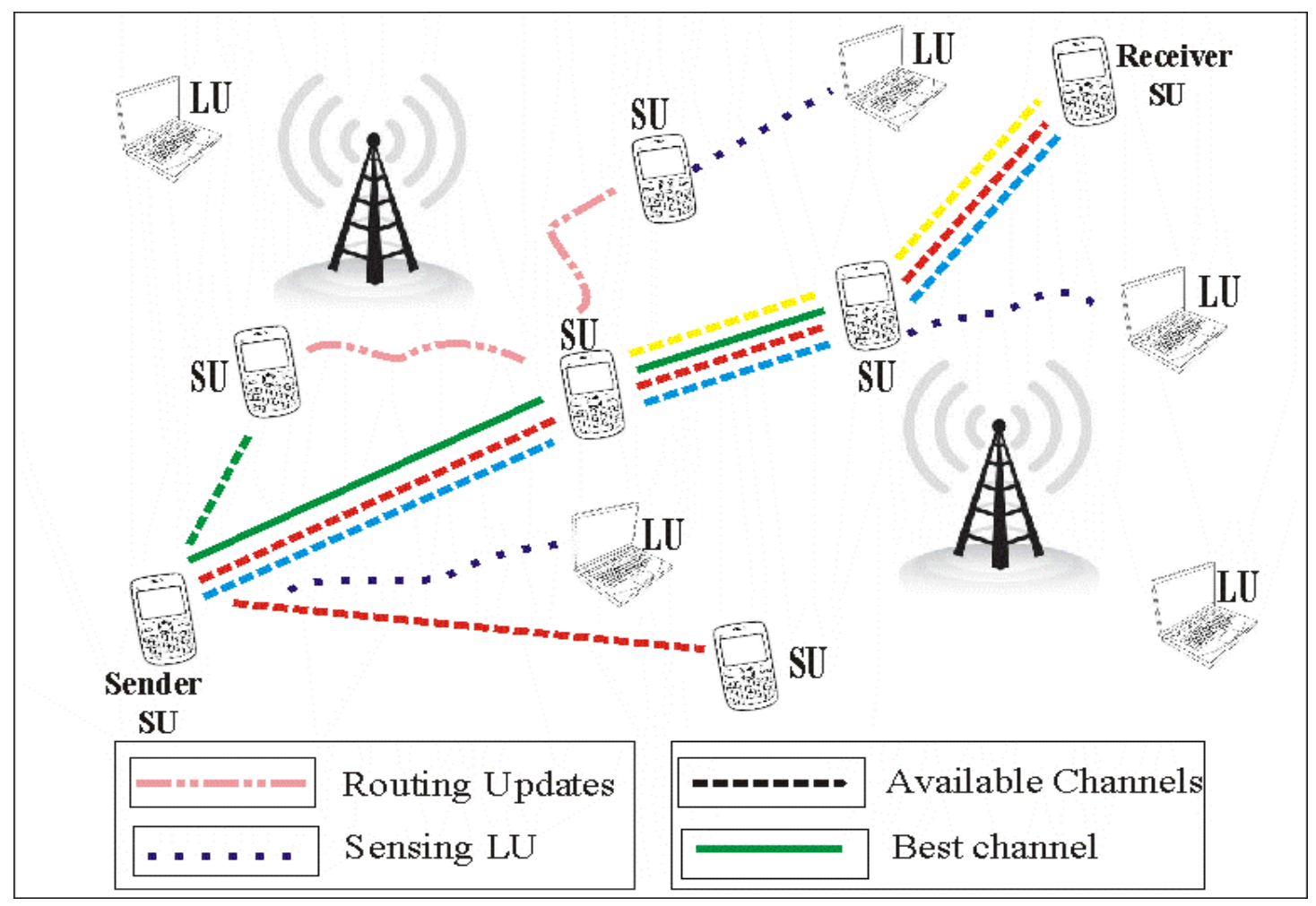

Fig 1: Example of the CRN and Multi-hop Routing

route is not only dependant on throughput, bandwidth, and delay but also on path stability and presence of multiple users (Primary or Secondary) competing for same Spectrum resource [3]. The dynamic spectrum that is intermittent in terms of both time and space keeps surrounding environment changing causes to reallocate the spectrum resources increasing communication overhead at every hop.

Dynamic Spectrum routing is challenging problem due to coexistence of both licensed and unlicensed users, in addition to that every node at each hop should be able to adapt to changing spectrum resource, learning about the spectrum occupancy and its history, making decisions on the quality of the available spectrum resource. Therefore it is essential to study the intelligent behaviours and interaction of multiple network users competing for spectrum resource.

In this paper we aim at providing detail insight into routing problem in multi-hope $\mathrm{CRN}$ in the context of dynamism due to intermittent time and space availability of spectrum. Moreover the dynamism which is also dependant on interaction between multiple users sharing same spectrum resource is also elaborated which is having great impact on route selection and spectrum management. Our aim is to give broader approach and give overall view of the field with focus on routing under consideration of multiple users sharing spectrum resource.

\section{ROUTING IN WIRELESS MULTI- HOP CRN}

The main objective of Routing in Multi-hop CRN is creation and maintenance of the route in open spectrum phenomenon. SUs can co-operate each other to form heterogeneous multi hop network across multiple primary networks. Multi-hop CRN consists of SUs relaying the information between sender and the receiver.
If physical capabilities of SUs are efficiently exploited it can sense, switch and transmit over many band. Unlike in multi radio multi-channel, multi-hop $\mathrm{CRN}$ have challenges of reducing the interference to PUs and manage intermittent spectrum resource.

SUs can operate under mixed environment in which they access unlicensed band or licensed bands. SUs can communicate with each other in two different ways i.e. through infrastructure in which they access secondary base-station and with each other in a multi-hop manner through ad-hoc connection on licensed or unlicensed band which is of our interest [4].

The basic problem statement of routing can be specified using the network model shown in figure 1. Wireless multi-hop network can be modelled as finite set of nodes consisting of $P$ number of PUs and $S$ number of SUs. There are $N$ orthogonal channels denoted by set $H$. Each node detects available channel and set of available channels are different from node to node [5]. $H_{i}$ and $n_{i}$ denotes the set and number of available channel at node $i$ respectively. Several spectrum bands are having different capacities and opportunities.

Each secondary node $i$ (where $1 \leq i \leq S$ ) has programmable radio interfaces denoted by $\rho_{\mathrm{i}}$. A radio interface is able to tune to a wide range of channels. For every link $l_{i j}$ between SU $i$ and $j$, has the set of available common channel $H_{l}$ which can be constructed by

$$
H_{l}=H_{i} \cap H_{j}
$$

Among all available common channels one optimal channel will be selected under the restriction of interference and fairness according to routing scheme to increase overall network throughput. Topology, channel availability and network dynamics are shared among all secondary nodes in the form of routing updates. Routing in wireless multi-hop network 
is deciding the path and intermediate/relay node with spectrum on each link from source to destination.

\section{ROUTING STRATEGIES AND PERFORMANCE EVALUATION CRITERIA'S}

Finding the appropriate path from source to destination in a network topology that evolves continuously is challenging problem. Under the consideration of network dynamics general routing strategies will not be able solve routing problem rather it is important to design strategy which will satisfy the secondary networks traffic demands and primary networks autonomy. Therefore design of routing protocol should consider different evaluation matrices according to the specific demand and network requirements of SUs [6].

Routing protocols can be categorized according to their performance and services given for unique challenges of CRN. Following three criteria's can effectively classify routing in multi-hop CRN:

\subsection{Functionality}

It refers to the functionality provided by routing protocols such as:

- Synchronization abilities of uncoordinated MAC protocol between two secondary nodes.

- Reduction in multiuser interference by avoiding high density regions.

- Link Reliability to reduce link instability.

- QoS requirement: Sufficient bandwidth for SU with minimum interference to PU

- Path selection which is not consisting of cycles

- Routing solution consisting of backup paths

\subsection{Efficiency}

It is the basic functionality requirement of routing protocol which can be given by:

- Network Throughput: This is a network capability to forward packets.

- End-to-end delay which is cumulative delay along the path from source to destination.

- Number of hops involved from source to destination

- Packet delivery ratio which is the probability of number of packets received at the destination.

\subsection{Suitability}

It is the ability of routing protocol to satisfy the application demand.

- Traffic Load taken by each link and node in CRN

- Time and space complexity of routing algorithm

- Route control complexity which is the number of control packets generated by every secondary node including sender and receiver.
- Overall protocol complexity can be given as aggregate time between route setup and final data transmission also considering rerouting.

- Protection mechanisms from security threats and unauthorized users

- Power control and energy consumption by every secondary node for routing and networking.

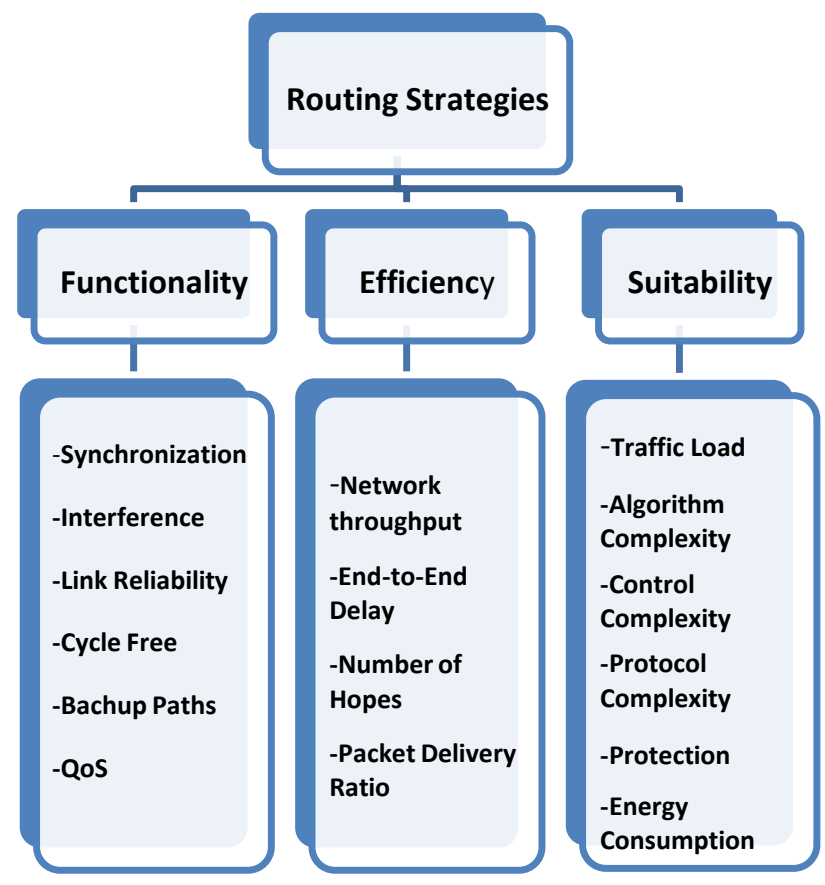

Fig 2: Routing Strategies

\section{ROUTING BASED ON FUNCTIONALITY}

\subsection{Interference temperature model based channel selection for end-to-end routing}

Static multi-hop multi-channel wireless mesh networks are considered for computation of available channels by each mesh node. Each mesh node measures the interference temperature for each channel locally and disseminates these measured values with other nodes within its interference range [7]. Based on the collected statistics all nodes compute a set of available channel for transmission.

Once available channels are computed it is required to select proper channel for transmission. Prerequisite of channel selection for link between two neighbouring nodes is that both the nodes must have non-empty intersection of their available channel sets i.e. for neighbouring node $m$ and $n$ to communicate:

$A C_{m} \cap A C_{n} \neq \varnothing$

It then uses per-hop link cost to select and end-to-end routing metric to select channels from available common channels set for each hop on the end-to-end path. Per-hop link cost is not only dependant on transmission delay and switching cost but 
also on channel stability factor which is average amount of time for which channel should remain available for transmission.

The routing metrics of end-to-end route $r$ consisting of $p$ hopes is formalized as

$$
R M(\mathrm{r})=(1-\delta) * \sum_{i=1}^{p} L C_{c i}+\delta *(1 \leq j \leq \mathrm{C} \times j)
$$

Route metrics $R M(r)$ takes first term as summation of $L C_{c i}$ which is cost of using channel $\mathrm{c}$ on hop $\mathrm{i}, \mathrm{Xj}$ is the total number of times channel $\mathrm{j}$ is used in route $\mathrm{r}$, and $\mathrm{C}$ is total number of channels in the spectrum pool. Link cost for channel $c$ is summation of Expected Transmission Time of frame $\left(\mathrm{ETT}_{\mathrm{c}}\right)$, Cost of Channel Switching $\left(\mathrm{SC}_{\mathrm{c}}\right)$ and Channel Stability Factor $\left(\mathrm{SF}_{\mathrm{c}}\right)$ given as,

$L C_{c}=E T T_{c}+S C_{c}+S F_{c}$

\subsection{Route Stability and Opportunistic routing}

Spectrum aware routing is necessary to balance between longterm route stability and short-term opportunistic performance. It selects routes with highest spectrum availability which is computed both the operations of the licensed spectrum users and the SU activities.

For achieving optimal routing it is necessary to concert work of Physical, MAC and Network layer. The spectrum aware optimal routing is defined as path with minimum hop count, increased end-to-end throughput and exploiting spectrum utilization.

SAMER in [8] creates forwarding mesh which is adjusted periodically according to the spectrum dynamics, and opportunistically routes packets across this mesh. The mesh created according to the long-term shortest path with minimum hop count but opportunistically expands or shrinks to exploit spectrum availability. It is built by computing for each node $i$ a cost Cost $_{i}$ which represent spectrum availability of the highest spectrum path whose length is less than $H$ hops. The value of the $H$ should complement with Cost $_{\max }$ which is the maximum cost to the destination.

\subsection{Cumulative interference and channel switching delay}

End-to-end communication in CRN is achieved by multiple channels therefore switching delay between channels must be considered for routing metrics [9]. Moreover intra and inter flow interference restrict the throughput and affect communication quality of CRNs. The intra flow interference is introduced by adjacent cognitive users and interflow interference is by users from neighbouring path.

Inter flow interference is reduced by selecting the appropriate transmission power in the physical layer. The SU's communication is interfered by the adjacent users in its interference range. The interference $I_{i, c}$ of secondary node $i$ is sum of interference from all users in its range on the same channel $c$,

$$
I_{i c}=\sum_{j \in S}^{n} I_{i c}
$$

The cumulative interference of the end-to-end connectivity at time $t$ can be described as:

$$
I(t)=\sum_{i c}^{\mathrm{N} 1, \mathrm{M} 1} \mathrm{I}_{\mathrm{ic}}(\mathrm{t}) \quad 1,2, \ldots, N 1, c=1,2, \ldots, M 1
$$

Where $\mathrm{N}_{1}$ is the number of $\mathrm{CR}$ users which include in the endto-end connectivity and $M_{1}$ is the number of channels. Minimum cumulative interference and channel switching delay helps in making decision of the optimal channel assignment and path selection.

\subsection{Topology control based routing}

When constructing network topology, topology control should take care of the interference from PU and the link availability. Prediction based cognitive topology control PCTC in [10] captures the network topology dynamically based on link prediction to provide efficient and opportunistic link management and routing.

This prediction of the link availability, duration can be used to construct a more reliable topology which results in reduced rerouting. Link available duration $\mathrm{T}_{\mathrm{a}}$ is:

$T_{a}=\min _{i=1,2 J \in\{P U s}\left\{T_{p} \times L\left(T_{p}\right), \hat{T}_{P i}^{j} \times L\left(\hat{T}_{P i}^{j}\right)\right\}$

The two ends of a link is given by $i$, and $j$ includes all the primary spectrum present in the network. The subscript $i$ and Superscript $j$ indicate that a link will be unavailable if any of its ends move into the interference region of any PU. The cognitive feature is enabled in $T_{a}$ due to its consideration of both node mobility and the interference to PU.

The principle of PCTC is to preserve the reliable path with maximum path weight for any pair of nodes under a connectivity guarantee. Topology construction process consist of three steps i.e. neighbor collection, path search and neighbor selection. The distributed localized Dijkstra topology control is used which aims at constructing an efficient topology which preserves the global network connectivity. It also preserves global reliable path between any two nodes i.e. it has a spanner factor of 1 regarding the path weight. PCTC results in longer path length but outperforms the predicted topology in terms of path duration.

\subsection{Backup channel and cooperative channel switching}

Network where nodes are equipped with single transceiver as in commodity wireless networks that run IEEE 802.11 DCF MAC does not provide synchronization of devices to single control channel. Therefore routing information should be exchanged in-band with data transmission on different frequency bands, which will introduce deafness problem.

Deafness occurs because of frequent channel switching in multichannel environment on single transceiver. Channel synchronization messages can reduce the deafness and hidden node problem. 
To optimize the routing performance and increase the network capacity, a cross layer routing and channel assignment which is not using any common control channel and accounts for the state of the link [11].

For avoiding end to end re-route procedure due to channel unavailability backup channel mechanism is used in which nodes are in advance aware of the channel to switch to in a situation where channel being currently used becomes unavailable due to PUs activity.

Each SU sends its prioritized channel information to neighbouring nodes on all available channels using message exchange. This information also include primary channel to be used for control and secondary as backup channel. Each node is aware in advance about two channels for communication. Secondary node handover and switch to the channel at their second priority list when a channel being used becomes unavailable. Cooperative channel switching is achieved by all the nodes by switching to backup channel. This cooperative behaviour solves all the problems related to channel heterogeneity and reliable exchange of the routing, channel and control information.

\subsection{End to end protocols evaluation for CRN}

The impact of the specific characteristics of CRN over TCP end-to-end performance such as sensing cycle, the interference caused by PU and channel heterogeneity should be investigated Comparison of two approaches of reactive routing protocols Single-path and Multi-path Routing is done in [12]. Due to dynamic nature of CRN wireless link may experience different conditions over time. Moreover sensing induced delay may increases route discovery frequency. Multi-path routing discovers multiple paths between Sources to Destination which are Node disjoint or Link disjoints. As alternate paths are available Multi-path routing significantly reduces route discovery frequency generated by each node. The performance of different TCP variants like TCP Reno, TCP NewReno, TCP selective acknowledgement and TCP Vegas are investigated over CRN.

\section{ROUTING WITH EFFICIENCY}

\subsection{Minimum Delay-Maximum stability Route through Opportunistic Service Differentiation}

To create minimum delay maximum stability route for end-toend traffic flow and to providing differentiated service to different traffic priorities defined by flow duration and flow priority [13]. The overall objective is achieved with the help of four different modules which Route Discovery, Route Decision, Opportunistic Routing and Route Maintenance.

Route Discovery node discovers all possible path between a source node and destination node. All the available paths are sorted according to delay which will be used by Route decision module to select path that can satisfy flow duration of the considered end-to-end traffic flow. After selection of the route opportunistic routing selects candidate forwarding node according to packet priority.

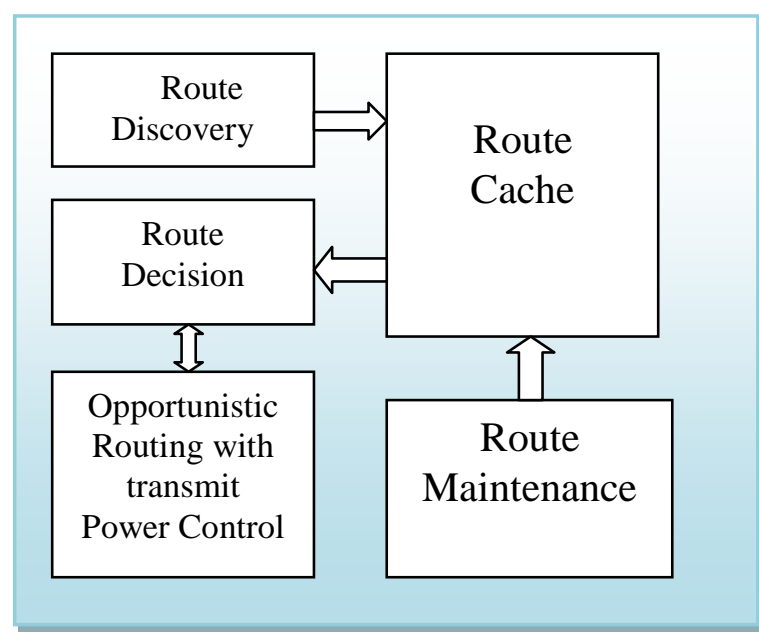

Fig 3: Opportunistic service differentiation routing protocol flow diagram

Higher priority packets have higher candidate selection range and higher chances of being received at further away nodes. Route maintenance module is responsible for deleting the stale entries from the route cache to keep size of it manageable.

\subsection{Spectrum-aware routing using forward and backward exploration}

The drawbacks like high latency time and high overhead of on demand routing is overcome using spectrum aware routing protocol [14]. In this scheme every node $\mathrm{x}$ stores table of $Q_{x}(y, d)$, which is an estimate for neighbour $y$ and destination $d$, of sum of number of channels available to destination through $x$. These values can be used to select best next hope and can be updated while routing using forward exploration. It is also clear that Dual Reinforcement Routing can improve the performance and time to learn good route with the help of forward and backward exploration. In a nutshell both of these algorithm exchange information with neighbours to adaptively learn good routes which have more available channels from just local information gradually incorporating more global state of the environment.

These algorithms outperform the spectrum aware shortest path routing but network sharing among competing operators is not considered which will affect spectrum availability at every hope.

\subsection{Cross layer joint opportunistic routing and dynamic spectrum sharing}

Distributed and localized algorithm for joint dynamic routing and spectrum allocation (ROSA) for multi-hop CRNs is taking decision based on locally collected spectrum and power allocation information $[15,16]$. Time slotted common control channel is used to collect network statistics and data channel for data communication. For reducing the probability of selecting congested intermediate relay node differential backlog is used which difference of queue backlog in given sessions. Feasible next hop is decided if it is having positive advance toward destination.

ROSA opportunistically calculates the next hop depending upon queuing and spectrum dynamics therefore each packet will select different path. If the destinations hop in the range of transmitter it will be selected directly. The transmitter may select a node other than destination if there is no available 
mini-band (low interference) between the transmitter and the destination.

\subsection{Joint spectrum-route selection with service differentiation}

Routing protocol can be designed to categorize routes according to requirement such as - Class I routes that provide better cognitive network performance, while the class II routes aims to achieve a higher measure of protection for PUs.

In class I route the CR network end-to-end latency is the key consideration where selected spectrum should support highest propagation distance and longest allowed duration for transmission [17]. The optimization finds the best spectrum $k$ out of $\mathrm{N}_{\mathrm{s}}$ possible spectrums bands to maximize the function:

$O_{\text {class }-I}=D_{k} \cdot \mathrm{T}_{\mathrm{f}}^{\mathrm{x}}$

Where $D_{k}$ specifies the distance covered for given transmission power and $T_{f}^{x}$ is fractional time for transmission considering different sensing schedule of the neighbouring users. This is interpreted as the as the highest number of packets may be transmitted over maximum possible distance.

The optimization function (equation -) for the Class II route is represented as the minimization of the product of the fractional area of overlap between the Cognitive and the PU ranges $A_{f}^{x}$ and the propagation distance $\mathrm{D}_{\mathrm{k}}$

$O_{\text {class }-I I}=D_{k} \cdot A_{f}^{x}$

The next hop selection is based on arriving route request packets at the destination indicating different routing paths. From this set it chooses the route with $\max \left\{\sum_{\forall j} O_{\text {class-I }}(\mathrm{j})\right\}$ or $\min \left\{\sum_{\forall j} O_{\text {class }-I I}(\mathrm{j})\right\}$ for class I and II routes, respectively.

The proactive route maintenance is achieved by $\mathrm{CR}$ user continuously monitoring its own location with respect to the known primary transmitter location. If the displacement of the node towards one or more PU is determined, it is beneficial to proactively discover the new path in case the current route fails

\subsection{Cumulative delay and node capacity based routing}

Control channel selection can be integrated with on-demand route discovery in high mobility multi-hop multichannel environments [18]. Clustering is used according to spectrum heterogeneity which is reducing the network scale and routing protocol overhead. The routing in multi-hop CRN includes intra-cluster and inter-cluster routing processes. The intracluster routing occurs in single cluster while inter-cluster routing occurs in multiple clusters. Due to high mobility nature, spectrum Route REQuest and Route REPly are used discover paths between nodes via the control channel message exchanges.

Route discovery scheme is integrated with node-importancebased clustering scheme and node contraction scheme. Since the high mobility nodes has adverse impact on data transmission due to a high link-disrupting rate, cluster formation and control channel selection schemes are beneficial to find out the stable routes to realize multi-hop connection
Proposed routing scheme integrates the on-demand routing with dynamic channel assignment. Since the path is determined by the largest path availability probability, the node's processing capacity determines the average end-to-end delay and packet delivery ratio for all data flows.

\subsection{Routing based on delay -Analysis}

Spectrum aware routing to utilize the spectrum resource in multi-hop single transceiver CRN (MSCRN) is proposed which is not using common control channel [19]. Link utilization is significantly improved by delay analysis.

Route Discovery: Any node initiates a route discovery by broadcasting the Route request packet on all available channels. Every intermediate node also transmits received packets on all available channels. The node state and working channel information of itself is also included when the node relay this Route request. So when node receives a route request it knows the working channels of nodes on the path route request is passing through. Every node is also having the knowledge of number of nodes on each channel. To reduce overhead of broadcasting route request on all available channels, number of available channels can be restricted to some threshold.

Route Reply: When destination node receives route request packet it assign a channel for connection establishment. Destination sends Route Reply to the source encapsulating the assigned channels on the working channel of pre-hop.

Switching node communication: Before switching to new node channel, the switching node broadcast a Special message LEAVE on current channel. After switching to new channel the switching node broadcast a JOIN messages on the new channel. Any node receiving LEAVE message from switching node stops transmitting on same channel until receiving JOIN message.

\subsection{Relay assisted routing}

Intermittent spectrum resource spectrum resource can be better matched to traffic demand of SUs with the help of relay node to assist the transmission and improve spectrum efficiency. The relay node may be selected for a link to bridge the links source and destination using its different common channel with those two nodes.

For improving end-to-end performance of the network Relay Assisted Protocol (RAR) is proposed in [20] which is usng link cost as performance metrics is given $b$ :

$\bar{C}_{i j}=\frac{1}{\overline{\mathrm{R}}_{\mathrm{ij}}}$

Where $\bar{C}_{\mathrm{ij}}$ is spectrum aware link cost and $\bar{R} \mathrm{ij}$ is potential capacity of link $(i, j)$ as:

$\bar{R}_{i j}=\sum_{n=1}^{k}\left(A_{i}^{k} A_{j}^{k} \frac{1-F_{i}^{k} F_{j}^{k}}{E T X_{i j}^{k}}\right)$

Link with more available channels, better channel condition, lower channel utilization will have smaller link cost, which will be more preferred by RAR routing protocol.

Potential relay nodes are within the common neighbour of the link's two end nodes,

$R_{i j}=N(i) \cap N(j)$ 
Where $N(i)$ stands for neighbours of $i$ and $N(j)$ stands for neighbours of $j$. Relay with maximum capacity will be selected among all neighbours if $i$ and $j$.

\section{SUITABILITY OF ROUTING SCHEMES}

\subsection{Relay based Routing with Energy consumption and Interference control}

Relaying is a promising solution to enhance the system capacity at low cost. In [21] author addressed the use of relays for routing in CRN with interference control. For deriving maximum reachable distance of SU in one-hop two conditions are evaluated.

- PU Transparency: The incurred interference does not interrupt the licensed user under QoS requirement.

- SU Reliability: The designated SU is able to successfully decode the data to meet its own QoS.

By reducing the transmit power of the SUs both of the above conditions are satisfied and channel quality is also maintained with shorter distance. This is achievable by multi-hop relaying with multiple short hops.

The relay route $\mathrm{SC}_{\mathrm{s}}-\mathrm{SC}_{1}-\mathrm{SC}_{2}-\ldots \ldots \ldots \ldots-\mathrm{SC}_{\mathrm{d}, \text {, where }} \mathrm{SC}_{\mathrm{s}}$ and $\mathrm{SC}_{\mathrm{d}}$ denoting source and destination respectively and $\mathrm{SU}_{\mathrm{i}}$ is the $\mathrm{i}^{\text {th }}$ intermediate relay node. These relay routes are providing an alternative path for direct route with single long hop $\mathrm{SC}_{\mathrm{s}}-\mathrm{SC}_{\mathrm{d}}$.

Multi-hop cooperative relaying is achieved through two routing algorithm, Nearest Neighbour Routing and farthest neighbour routing. Nearest neighbour routing attempt to find nearest neighbour inside the sector improves channel quality and saves energy consumption at every node.

Farthest neighbour routing attempt to find farthest neighbour with in the sector consisting of few long hops in the complete path which is good for reducing the delay but tend to consume more energy to achieve good channel quality.

\subsection{Routing based on Spectrum availability and Load estimation}

Distinguishing features of a multi-hope CRN emphasize on efficient routing algorithm which will offer contemporaneous end to end path. Routing protocol for Cognitive Radio Adhoc Network should incorporate two routing matrices, spectrum availability cost and Load Estimation [22].

Spectrum availability cost is used to track link connectivity behaviour and assign persistent cost metric which is periodically updated to reflect its overall behaviour. Link with minimum interfering times will be selected at every hope. If the nodes finds next hop for the route causing interference to the PUs, it buffers the packet for predetermined short time eventually forward it when link become available. Moreover, any change in the link state database triggers a fresh next hope route computation. Every CR node is also maintaining traffic information going through itself to select the least loaded path for new communication. In case of unavailability of the next hop route copy of the message is also send to other neighbouring nodes if the spectrum availability cost is lower at these nodes.

\subsection{Accelerating routing convergence with forward and backward ants}

An artificial ANT colony system can be used for discovering, observing and learning of routing strategies by guided ants communicating in an indirect way [23].

Local heuristic information and statistical history information is maintained by each node. Common spectrum between node $k$ and neighbour node $i$ can be characterized as local heuristic information $l_{i}^{k}$. Statistics of the history path quality between $k$ and $j$ is represented using $\mu_{j}^{k}$ and $\tau_{i j}^{k}$ indicates the preference of selecting node $i$ when destination is $j$.

Every node exchanges information with all its neighbours about current spectrum availability using common control channel. For finding and maintaining path to any destination forward ant is generated and sent in broadcast way to its neighbours if no routing information is known to reduce route discovery time. Otherwise node transmits forward ants in stochastic unicast way.

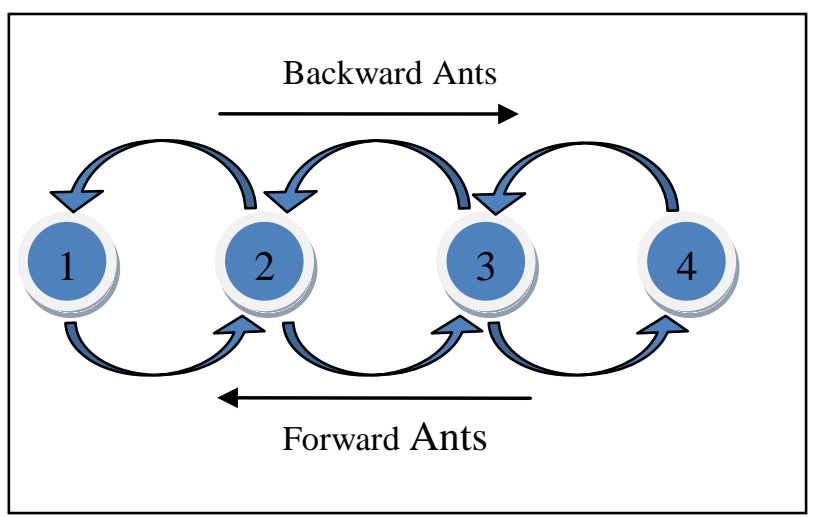

Fig 4: Information exchange using Ants

The probability of of node $k$ chooses $i$ as next hope for destination $d$ is:

$\operatorname{Prob}_{i d}^{k}=\frac{\left(\tau_{i d}^{k}\right)^{\alpha} \cdot\left(l_{i}^{k}\right)^{\beta}}{\sum_{i \in N_{e i}(k)}\left(\tau_{i d}^{k}\right)^{\alpha} \cdot\left(l_{i}^{k}\right)^{\beta}}$

Where $\alpha$ and $\beta$ represent the respective adjustable weights of pheromones concentration and local heuristic. Comparative Higher value of $\beta$ strengthens the forward ant's ability to explore better path. Node $j$ on receiving forward ant from node $i$ check the value $l_{i}^{j}$ in routing table. If common data channels exist between $i$ and $j$, node $j$ puts its address into forward ant and forward it to its neighbours. Forward ant travel and records a path that is spectrum feasible.

On reaching the forward ant at the destination backward ant is generated tracking record of local heuristic information and update routing table while travelling along their paths. Path's relative goodness is considered for accelerating convergence.

\subsection{Routing under consideration of environment dynamics}

In multi-hop CRN, the topology and connectivity maps are determined by the available spectrum holes and their instantaneous variations. Routing solution considering 
activities of PU and holding time of the exploited primary bands is proposed in [24].

According to the PUs activity over the primary channels cognitive environment is classified in to three categories:

- Static: Holding time offers relatively static wireless environment, Once frequency band is available it can be exploited for an unlimited period of time.

- Dynamic: The intermittent availability of the exploited spectrum band seriously affects the services offered to the SU.

- Highly Dynamic: Due to highly active PUs, spectrum bands are not available for whole communication duration. Possible solution is to opportunistically transmit over any available channel.

In static multi-hop primary frequency band is available for a duration that exceeds the communication time in which optimal routing can be derived depending upon traffic demands, available channel capacity and mesh router positions i.e. topology and traffic throughput. Routing design in this scenario should consider detection of the new arriving primary nodes over the exploited bands and which reaction will be taken Routing design should account for intra cognitive node interference and PU interference.

In order to find available and stable path in dynamic CRN issues like route stability, exchanging control information and channel synchronization should be handled carefully. Routing should be assisted by a metric from lower layer which should reflect the spectrum availability and its quality.

Centralized synchronization window can be used for sharing control information, which consists of fixed time slot where all the nodes are tuned to all the frequencies and exchange all possible control information. The control information can also be shared on low frequency common control channel. In highly dynamic environment a complete opportunistic solution can be opted where every packet can be sent and forwarded over opportunistically available channels constitutes potential solution.

\subsection{Cooperative price-based relay routing}

The benefits of game theory and optimal methods are leveraged in [25] for proposing efficient solutions of competitive pricing and marketing model problem for spectrum sharing and allocation.

Hierarchical spectrum sharing network (HSSN) consisting of infrastructure based and Ad-Hoc cells is combined with economic paradigm to form spectrum utility maximization framework by prizing the spectrum used for communicating with different destinations among a number of SUs and base station of one cell. Base station decides the prices of the spectrum each SU uses for transmission as a decision maker. Each SU makes an allocation of its spectrum itself and according to its utility maximization decides the next hope transmitted to.

\subsection{IP hop-by-hop geographic spectrum aware routing}

Every secondary node in CRN must know the number of channels, identity and offered quality to allocate resources for data transmission. IP spectrum aware routing protocol takes all mentioned information from lower two layers sensing results [26].

The data packets header is with information needed by each node to take local decision based on its local neighbourhood's information regarding the next hop to forward the packets towards the destination. The intersection of channels sensed by two nodes (Current node and next hope node), find the jointly sensed channels. Good quality of service (QoS) channel can be selected from this intersection.

IPSAG uses the local information i.e. position and channels opportunities over neighbourhood and takes hop-by-hop geographic routing decision with QoS. The criterion used for creation of neighbourhood is the Euclidian distance which can be represented by circle with the core given by the corresponding node and the radius given by the maximum Euclidian distance between the core and secondary node as shown in fig.5.

If a packet encounters preexisting systems like WiMAX or WiFi it will be routed according to the system's way of routing, based on the tunneling routing approach, otherwise Secondary node route according to IPSAG.

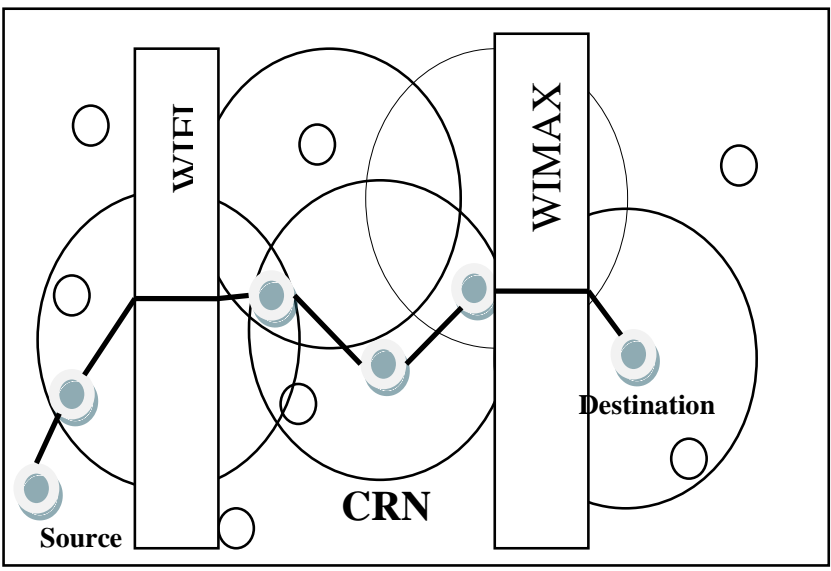

Fig 5: Routing with Euclidian Distance and tunnelling routing

\subsection{Connectivity based routing scheme}

Connectivity in CRN is significantly affected by PU's behaviour. In this view, a new distributed protocol named Gymkhana is proposed in [27] which is able to measure the connectivity of different network paths and to route data packets across paths that avoid network zones that do not guarantee stable and highly connected links.

To discover all possible paths towards the destination source node broadcast a route request packet across the network. The destination uses the information contained in the received route request to run the Gymkhana algorithm to classify paths on the basis of their connectivity and to select one path among all discovered paths.

At the destination contents of the route request are processed in two steps:

1. Virtual graph is formed for all paths. 
2. Calculation of the eigen values of the Laplacian of the virtual graph which is used to evaluate the connectivity of a path.

Proposed mathematical model evaluates the connectivity of the different paths by taking into account the PUs behaviour, the cost of channel switching and the hop count.

\section{DISCUSSION AND FUTURE DIRECTIONS}

In this paper, we present an overview of the state of the art for routing protocol in CRN. The existing work in multi-hop CRN and routing is discussed. We defined the distinctive features of the multi-hop CRN explicitly endorsing network dynamics and variability. Problem of routing and its unique challenges are defined by taking the dynamic environment in to account.

For analytical evaluation of the routing protocol we divided the routing strategies in three main categories i.e. routing with special functionality, efficiency and suitability to particular network requirement which is briefed in table 1. This categorization is according to performance and services of routing protocol given for unique challenges of CRN.

With respect to routing in CRN we believe that there is future work needed in devising accurate model that accounts for online environment. Every participating SU needs a set up for learning and strategy- choosing along the optimal path selection from source to destination.

We strongly believe that there is significant scope for devising routing protocol that adopts the instantaneous variations in the network environment and incomplete information. To this extent we believe that routing in multi-hop CRN is open research issue and need to be addressed in more detailed manner. Below, we summarise some open research issues in modelling and designing CRN:

\subsection{Exchanging routing information}

Many routing solution currently use common control channel for sharing routing updates which are useful in neighbour discovery, route discovery and route establishment. Due to the fact that SU is using spectrum as visitor common control channel can also be affected by PUs activity. Moreover, selection of one particular channel as global control channel seriously affects network performance.

Alternate methods for sharing information can be local and global broadcast which are used by traditional routing protocols. As all nodes are tuned to different channel broadcasting must be done on all available channel to find out common channels for transmission which naturally increase protocol overhead.

In highly mobile and dynamic environment, information exchange is only means of creating view of overall current topology of network. Design and modelling of the solution considering above fact will really improves the performance of routing.

\subsection{Dynamic topology and unstable connectivity}

Due to high mobility of the SUs or PU activities topology and connectivity of the network is significantly affected. The reachable neighbours of the node may change their location due to mobility or they will not be used as relaying node as PUs start exploiting network.

Solution based on static environment and stable topology will not serve the purpose of route selection in intermittently connected network. In Addition to this routing should be reactive which is taking in to account the rapid changes in the neighbouring table and accordingly next hop selection must be done. Moreover, due to PUs activities the common channel list for transmission may changes between different nodes. As a result same neighbour can be viewed with another common channel. Changes in resource availability may affect the network throughput due to resource reallocation.

In addition to above discussed scenario it is possible that common channel list becomes empty which results in new next hope selection affecting the network end to end delay and throughput.

We strongly believe that design of routing protocol should consider intermittent availability of the spectrum both in terms of time and space which remains very important research issue

\subsection{Reconfiguration of routing path}

Due dynamic topology and intermittent spectrum availability selected path becomes unusable in particular area resulting in route failure. Reconfiguration of the routing path by selecting new nodes or different channel for communication can solve this problem.

Naturally reconfiguration of paths increases the protocol overhead therefore the routing algorithm should be spectrum aware to reduce rerouting. By spectrum aware we mean that routing algorithm should learn good route and bad route from previously taken decision.

Spectrum fluctuations, channel stability and history should be considered as important metrics in route selection. Moreover predictions of channel stability and link quality in future will really helpful in design of effective routing solution.

To this end we suggest that, apart from convergence and spectrum aware properties of routing algorithm, it should be designed to boost the performance by using machine learning and prediction tools. The potential benefits of learning from the previous experience will surly helps in controlling reconfiguration overhead.

\subsection{PUs behaviour}

The PU behaviour is a key parameter for taking routing decision in multi-hop CRNs. The autonomy of the PU will naturally affected by the presence of the SU. The ideal behaviour of SU vacating the spectrum upon detection of primary activity is not expected in all networks.

Practically fully cooperative behaviour from PU cannot be taken for granted. Instead, PUs will only cooperate with SUs if the cooperation can bring them some benefits [28] Otherwise they will ensure that no SU should be able to use primary spectrum.

To tackle the above challenge it really important to study, model and analyse the PU's behaviour which results in designing efficient, self enforcing routing scheme. Spectrum leasing or voluntary spectrum handoff from PUs can be few example of spectrum management which can be useful in taking efficient routing decisions. 
Table. 1. Routing strategies Summarization

\begin{tabular}{|l|c|l|c|l|c|}
\hline \multicolumn{7}{|c|}{ Routing strategies } \\
\hline Functionality & Protocol & \multicolumn{1}{|c|}{ Efficiency } & Protocol & Suitability & Protocol \\
\hline Synchronization & {$[10]$} & Network throughput & {$[14,17,20]$} & Traffic Load & {$[22]$} \\
\hline Interference & {$[7,9,12,17]$} & End-to-End delay & {$[13,18,19]$} & Algorithm Complexity & {$[23]$} \\
\hline Link reliability & {$[8]$} & Number of hopes & {$[15,16,20]$} & Control Complexity & {$[24]$} \\
\hline QoS & {$[12]$} & Packet delivery ratio & {$[18]$} & Protocol Complexity & {$[22,25,26]$} \\
\hline Backup Paths & {$[11]$} & & & Protection & {$[27]$} \\
\hline & & & & Energy Consumption & {$[21]$} \\
\hline
\end{tabular}

The importance of studying the PU's behaviour will help in modelling the spectrum sharing among network users with various optimality criteria.

\subsection{SUs behaviour}

CRN are equipped with intelligent users who are able to observe learn and act to optimize their performance. Fully cooperative behaviour of SU with each other cannot be taken for granted if they belong to different authorities and pursue different goals. Multiple SUs compete non-cooperatively and independently with each other for spectrum sharing. They will only cooperate with each other if the co-operation can bring them more benefits.

The optimization of spectrum usage among multiple users is generally a multi-objective optimization problem, which can be studied with different constituents and behaviour of the SUs. The interaction among multiple SUs can be cooperative, Non cooperative, stochastic or Economic. The comprehensive study of this can efficiently improves the routing performance for multiple SUs along the routing path.

Spectrum sharing among multiple SU at every hop along the path can be improved by jointly studying their behaviour by leveraging game theory and multi-agent learning [29, 30]. The main challenge in multi-agent learning is that each learning agent must explicitly consider other learning agents, and coordinate their behaviour with theirs, such that coherent joint behaviour results.

\section{CONCLUSIONS}

Routing in CRN is a young, but active and rapidly exploring field of research. It integrates results of spectrum management and spectrum aware routing. CRN necessitates protocol enabling the design of algorithm that learns the solution to nonlinear, stochastic routing task about which it possesses limited knowledge.

In this comprehensive survey, we have discussed in detail the problem of routing and its unique challenges in online stochastic environment together with promising ways of addressing these issues. A classification of different routing strategies is given with respect to their functionality, efficiency and suitability.

Regard the existing routing solutions, several open issues remain that must be addressed. Firstly, the information from multiple users must be seamlessly integrated in the working of routing protocol. Most of the existing works do not completely integrate the routing with network user behaviour. PU's behaviour and SU's interaction should be considered as important routing design issue.
Many avenues for Routing in multi-hop CRN are open at this point and many research opportunities presents themselves. In particular, this survey strongly recommends spectrum aware routing protocols that consider the interaction and behaviour of all network users.

In our view, significant progress in the field of routing in multihop CRN can be achieved by a more intensive cross fertilization between the fields of dynamic decision theories and multi-agent learning.

\section{REFERENCES}

[1] Mitola, J., III; Maguire, G.Q., Jr.; , "Cognitive radio: making software radios more personal," Personal Communications, IEEE , vol.6, no.4, pp.13-18, Aug 1999 doi: $10.1109 / 98.788210$.

[2] Ian F. Akyildiz, Won-Yeon Lee, Mehmet C. Vuran and Shantidev Mohanty, Next generation/dynamic spectrum access/cognitive radio wireless networks: A survey, International Journal on Computer Networks (Elsevier), Vol. 50, 2006, pp. 2127-2159.

[3] Matteo Cesana, Francesca Cuomo and Eylem Ekici, Routing in cognitive radio networks: Challenges and solutions, International Journal on Ad Hoc Networks (Elsevier), Vol. 9, Issue 3, May, 2011, pp. 228-248

[4] Cormio C, K.R. Chowdhury, A survey on MAC protocols for cognitive radio networks, Ad Hoc Networks 7 (7) (2009) 1315-1329, doi:10.1016/j.adhoc.2009.01.002.

[5] Jian Tang; Hincapie, R.; Guoliang Xue; Weiyi Zhang; Bustamante, R.; , "Fair Bandwidth Allocation in Wireless Mesh Networks With Cognitive Radios," Vehicular Technology, IEEE Transactions on , vol.59, no.3, pp.14871496, March 2010,doi: 10.1109/TVT.2009.2038478

[6] Long Zhang; Xianwei Zhou; Huayi Wu; , "A rough set comprehensive performance evaluation approach for routing protocols in cognitive radio networks," Global Mobile Congress 2009 , vol., no., pp.1-5, 12-14 Oct. 2009 doi: 10.1109/GMC.2009.5295841

[7] Sharma, M.; Sahoo, A.; Nayak, K.D.; , "Channel Selection Under Interference Temperature Model in Multi-Hop Cognitive Mesh Networks," New Frontiers in Dynamic Spectrum Access Networks, 2007. DySPAN 2007. 2nd IEEE International Symposium on, vol., no., pp.133-136, 17-20 April 2007,doi: 10.1109/DYSPAN.2007.25. 
[8] I. Pefkianakis, S. Wong, S. Lu, SAMER: spectrum aware mesh routing in cognitive radio networks, in: 3rd IEEE Symposium on New Frontiers in Dynamic Spectrum Access Networks, DySPAN 2008, 2008, pp. 1-5, doi: 10.1109/DYSPAN.2008.90

[9] Jiao Wang; Yuqing Huang; , "A cross-layer design of channel assignment and routing in Cognitive Radio Networks," Computer Science and Information Technology (ICCSIT), 2010 3rd IEEE International Conference on , vol.7, no., pp.542-547, 9-11 July 2010 doi: 10.1109/ICCSIT.2010.5564800.

[10] Quansheng Guan; Yu, F.R.; Shengming Jiang; Gang Wei; , "Prediction-Based Topology Control and Routing in Cognitive Radio Mobile Ad Hoc Networks," Vehicular Technology, IEEE Transactions on , vol.59, no.9, pp.44434452, Nov. 2010,doi: 10.1109/TVT.2010.2069105

[11] Zeeshan, M.; Manzoor, M.F.; Qadir, J.; , "Backup channel and cooperative channel switching on-demand routing protocol for multi-hop cognitive radio ad hoc networks (BCCCS)," Emerging Technologies (ICET), 2010 6th International Conference on , vol., no., pp.394-399, 18-19 Oct. 2010,doi: 10.1109/ICET.2010.5638455

[12] Marco Di Felice, Kaushik Roy Chowdhury, Wooseong Kim Andreas Kassler and Luciano Bononi, End-to-end protocol for cognitive Radio Ad Hoc Networks: An evaluation study, International Journal on Performance evaluation (Elsevier), Article in Press, November 2010.

[13] Kiam Cheng How, Maode Ma and Yang Qin, Routing and QoS provisioning in cognitive radio networks, International Journal on Computer Network (Elsevier), Vol. 55, Issue 1, January 2011. pp 330-342.

[14] Bing Xia; Wahab, M.H.; Yang Yang; Zhong Fan; Sooriyabandara, M.; , "Reinforcement learning based spectrum-aware routing in multi-hop cognitive radio networks," Cognitive Radio Oriented Wireless Networks and Communications, 2009. CROWNCOM '09. 4th International Conference on , vol., no., pp.1-5, 22-24 June 2009, doi: 10.1109/CROWNCOM.2009.5189189.

[15] L. Ding, T. Melodia, S. Batalama, M.J. Medley, Rosa: distributed joint routing and dynamic spectrum allocation in cognitive radio ad hoc networks, in: MSWiM '09: Proceedings of the 12th ACM International Conference on Modeling, Analysis and Simulation of Wireless and Mobile Systems, 2009, pp. 13-20, doi: 10.1145/ 1641804.1641810.

[16] Lei D, Tommaso M, Stella N. B,J. D. Matyjas, and M. J. Medley, Cross-Layer Routing and Dynamic Spectrum Allocation in Cognitive Radio Ad Hoc Networks, IEEE TRANSACTIONS ON VEHICULAR TECHNOLOGY, VOL. 59, NO. 4, MAY 20101969

[17] Chowdhury, K.R.; Akyildiz, I.F.; , "CRP: A Routing Protocol for Cognitive Radio Ad Hoc Networks," Selected Areas in Communications, IEEE Journal on , vol.29, no.4, pp.794-804, April 2011, doi: 10.1109/JSAC.2011.110411

[18] Xin-Lin Huang; Gang Wang; Fei Hu; Kumar, S.; , "Stability-Capacity-Adaptive Routing for High-Mobility Multihop Cognitive Radio Networks," Vehicular Technology, IEEE Transactions on , vol.60, no.6, pp.27142729, July 2011, doi: 10.1109/TVT.2011.2153885

[19] Huisheng Ma; Lili Zheng; Xiao Ma; Yongjian luo; , "Spectrum Aware Routing for Multi-Hop Cognitive Radio Networks with a Single Transceiver," Cognitive Radio
Oriented Wireless Networks and Communications, 2008. CrownCom 2008. 3rd International Conference on , vol., no., pp.1-6, 15-17 May 2008, doi: 10.1109/CROWNCOM.2008.4562509

[20] Juncheng Jia; Jin Zhang; Qian Zhang; , "Relay-Assisted Routing in Cognitive Radio Networks," Communications, 2009. ICC '09. IEEE International Conference on , vol., no., pp.1-5, 14-18 June 2009, doi: 10.1109/ICC.2009.5199406

[21] Min Xie; Wei Zhang; Kai-Kit Wong; , "A geometric approach to improve spectrum efficiency for cognitive relay networks," Wireless Communications, IEEE Transactions on , vol.9, no.1, pp.268-281, January 2010, doi: 10.1109/TWC.2010.01.090180.

[22] Talay, A.C.; Altilar, D.T.; , "ROPCORN: Routing protocol for cognitive radio ad hoc networks," Ultra Modern Telecommunications \& Workshops, 2009. ICUMT '09. International Conference on , vol., no., pp.1-6, 12-14 Oct. 2009, doi: 10.1109/ICUMT.2009.5345349.

[23] Bowen Li; Dabai Li; Qi-hui Wu; Haiyuan Li; , "ASAR: Antbased spectrum aware routing for cognitive radio networks," Wireless Communications \& Signal Processing, 2009. WCSP 2009. International Conference on, vol., no., pp.1-5, 13-15 Nov. 2009, doi: 10.1109/WCSP.2009.5371704

[24] Khalife, H.; Malouch, N.; Fdida, S.; , "Multihop cognitive radio networks: to route or not to route," Network, IEEE, vol.23, no.4, pp.20-25, July-August 2009 doi: 10.1109/MNET.2009.5191142.

[25] Nan Li; Lei Gong; Shaoqian Li; , "Price-based spectrumallocation relay routing in cognitive radio networks," Information, Communications and Signal Processing, 2009. ICICS 2009. 7th International Conference on , vol., no., pp.1-5, 8-10 Dec. 2009, doi: 10.1109/ICICS.2009.5397471.

[26] Bădoi, C.-I.; Croitoru, V.; Prasad, R.; , "IPSAG: An IP Spectrum Aware Geographic Routing algorithm proposal for multi-hop Cognitive Radio networks," Communications (COMM), 2010 8th International Conference on , vol., no., pp.491-496, 10-12 June 2010 doi: 10.1109/ICCOMM.2010.5509020

[27] A. Abbagnale, F. Cuomo, Gymkhana: a connectivity-based routing scheme for cognitive radio ad hoc networks, in: IEEE Conference on Computer communications, INFOCOM 2010, pp. 1-5, doi:10.1109/ INFCOMW.2010.5466618.

[28] Beibei Wang, Yongle Wu and K.J Ray Liu, Game theory for Cognitive Radio networks: an Overview, International Journal on Computer Network s (Elsevier), Vol. 54, Issue 14, October 2010, pp 2537-2561.

[29] Rajneesh Sharma and M. Gopal, Synergizing reinforcement learning and Game Theory-A new direction for control, International Journal Applied Soft Computing 10 (Elsevier), 2010, pp. 675-688.

[30] LIU Wei-bing and WANG Xian-jia, Dynamic Decision Model in Evolutionary Games Based on Reinforcement Learning, International Journal on System EngineeringTheory \& Practice, Vol. 2, Issue 3, Feb 2009,pp 28-33 